\title{
Impact of percutaneous coronary intervention timing on 5-year outcome in patients with non-ST-segment elevation acute coronary syndromes. The 'wait a day' approach might be safer
}

\author{
Erik F.J. Oosterwerff ${ }^{1}$ N.D. Fagel ${ }^{1}$ T. Slagboom ${ }^{1}$ J.G. P. Tijssen ${ }^{2} \cdot$ J.P. Herrman ${ }^{1}$ $^{2}$ \\ P.C. Smits ${ }^{3} \cdot$ M.J. Suttorp ${ }^{4}$ E. Ronner ${ }^{5} \cdot$ G.J. Laarman ${ }^{6} \cdot$ M.S. Patterson ${ }^{1}$ \\ G. Amoroso ${ }^{1}$ - M.A. Vink ${ }^{1}$ - R.J. van der Schaaf ${ }^{1} \cdot$ F.W.A. Verheugt ${ }^{1} \cdot$ R.K. Riezebos ${ }^{1}$
}

Published online: 9 February 2016

(C) The Author(s) 2016. This article is published with open access at Springerlink.com

\begin{abstract}
Background The OPTIMA trial was a randomised multicentre trial exploring the influence of the timing of percutaneous coronary intervention (PCI) on patient outcomes in an intermediate to high risk non-ST-elevation acute coronary syndrome (NSTE-ACS) population. In order to decide the best treatment strategy for patients presenting with NSTEACS, long-term outcomes are essential.

Methods Five-year follow-up data from 133 of the 142 patients could be retrieved $(94 \%)$. The primary endpoint was a composite of death and spontaneous myocardial infarction (MI). Spontaneous MI was defined as MI occurring more than 30 days after randomisation. Secondary endpoints were the individual outcomes of death, spontaneous MI or re-PCI. Results No significant difference with respect to the primary endpoint was observed (17.8 vs. $10.1 \%$; HR 1.55, $95 \%$ CI: $0.73-4.22, p=0.21$ ). There was no significant difference
\end{abstract}

Erik F.J. Oosterwerff

erikoosterwerff@hotmail.com

1 Heart Centre, Department of Cardiology, Onze Lieve Vrouwe Gasthuis, 1090 HM PO Box 95500, Amsterdam, The Netherlands

2 Heart Centre, Department of Cardiology, Academic Medical Centre, Amsterdam, The Netherlands

3 Heart Centre, Department of Cardiology, Maasstad Hospital, Rotterdam, The Netherlands

4 Heart Centre, Department of Cardiology, St Antonius Hospital, Nieuwegein, The Netherlands

5 Heart Centre, Department of Cardiology, Reinier de Graaf Hospital, Delft, The Netherlands

6 Heart Centre, Department of Cardiology, TweeSteden Hospital, Tilburg, The Netherlands in mortality rate. However, spontaneous MI was significantly more common in the group receiving immediate PCI (11.0 vs. $1.4 \%$; HR $4.46,95 \%$ CI: $1.21-16.50, p=0.02$ ). We did not find a significant difference between the groups with respect to re-PCI rate.

Conclusion There was no difference in the composite of death and spontaneous MI. The trial suggests an increased long-term risk of spontaneous MI for patients treated with immediate PCI.

Keywords NSTE-ACS - Timing of PCI - Long-term outcomes

\section{Introduction}

The optimal timing when using percutaneous intervention $(\mathrm{PCI})$ in a non-ST-elevation acute coronary syndrome (NSTE-ACS) is still a matter of much debate. Early intervention may be beneficial in patients with NSTE-ACS as it could prevent a new myocardial infarction (MI). On the other hand, it could prove more harmful as it might lead to an increased incidence of periprocedural complications due to the thrombotic tendency of the culprit lesion. First-line medical pacification of the culprit coronary plaque may be beneficial but it requires time, during which a recurrent infarction may occur. The current guidelines recommend urgent (ESC guidelines definition: $<2 \mathrm{~h}$ ) coronary angiography in patients with a very high risk, defined as: refractory angina, with associated heart failure, life-threatening ventricular arrhythmias, or haemodynamic instability. In addition, early (ESC guidelines definition: $<24 \mathrm{~h}$ ) coronary angiography is recommended in patients with a GRACE risk score above 140 or with high-risk features, defined as a relevant rise or fall in troponin or dynamic ST or T-wave changes $[1,2]$. 
The optimal timing of angiography and revascularisation in NSTE-ACS has been studied quite extensively. Yet the influence of the timing on PCI remains less clear due to the fact that previous trials chose to randomise the timing of the coronary angiography. The OPTIMA trial randomised patients with a suspected NSTEMI into immediate or delayed PCI after their eligibility had been established by acute coronary angiography [3].

In contrast to trials comparing routine versus selective invasive intervention, no long-term data from trials evaluating optimal timing of angiography have been published. Results of trials investigating routine versus selective invasive intervention in NSTE-ACS suggest that spontaneous $\mathrm{MI}$ in contrast to periprocedural MI increases long-term mortality [4-6]. Long-term outcomes should be known in order to decide the proper treatment strategy for patients presenting with NSTE-ACS.

In the current study, the 5-year clinical outcomes of death, MI and revascularisation of the OPTIMA trial are reported.

\section{Materials and methods}

\section{Study design}

The original study design and the results have been published previously [3] and the protocol was approved by the review board of all the participating centres. All patients gave informed consent.

The OPTIMA trial was a randomised multicentre trial exploring the influence of the timing of PCI on patient outcomes in an intermediate to high risk NSTE-ACS population. Follow-up was done on all the patients who had given their informed consent in the original OPTIMA trial. In cases where no information regarding the patient's vital status or above-mentioned endpoints at 5-year follow-up could be retrieved from the patient file, the general practitioner and/or the local authorities were contacted. After it had been confirmed that the patient was still alive, the patient was contacted by telephone.

\section{Outcomes}

The main outcome in the analysis was the combined endpoint of death and spontaneous MI. The secondary endpoints were the individual outcomes of death, spontaneous MI or re-PCI. Spontaneous MI was defined as: MI occurring more than 30 days after randomisation. The universal definition for MI was used [7]. The definition includes the detection of a rise and/ or fall of troponin $\mathrm{T}$ with at least one value above the 99th percentile upper reference limit and with at least one of the following: symptoms of ischaemia, new or presumed new significant ST-segment-T wave (ST-T) changes or new left bundle branch block (LBBB), development of pathological Q waves on the ECG, imaging evidence of new loss of viable myocardium or new regional wall motion abnormality.

\section{Statistical analysis}

The analysis was based on the intention to treat. Cumulative event rates were estimated using the Kaplan-Meier method. Hazard ratios (HRs) with $95 \%$ confidence intervals (CIs) were obtained with Cox proportional hazards models and included the treatment strategy as the only covariate. Pre-specified subgroup analyses included gender, smoking, hypertension and diabetes. The GRACE RISK score was calculated for stratification purposes in all patients. Statistical analyses were applied using the Statistical Package for Social Sciences (SPSS version 15.0 for Windows, Chicago, Illinois).

\section{Results}

From March 2005 until April 2007, 251 patients were enrolled in the OPTIMA trial. After undergoing immediate angiography, a culprit lesion amenable to PCI was identified in 142 patients. Patients were not randomised when angiography did not demonstrate significant coronary stenosis amenable for PCI, when coronary artery bypass grafting (CABG) was judged to be the preferred treatment, when the culprit lesion was instent restenosis or if the affected myocardial territory involved a chronic occlusion. These patients were treated according to institutional practices. The study randomised the patients amenable to PCI to an immediate $(n=73)$ or deferred $(n=69)$ coronary intervention. Table 1 shows the baseline characteristics, which were similar in the two groups, except for more previous $\mathrm{CABG}$ in the immediate group (11 vs. $1 \%$, $p=0.02)$ and the increased prevalence of hypertension in the immediate group ( 53 vs. $33 \%, p=0.03$ ). The results of 30 -day and 6-month follow-up were published previously [3] in which $74(52 \%)$ of the 142 patients showed multivessel disease. Of these, $26 \%$ underwent treatment of two or more lesions leading to complete revascularisation in $74 \%$ of the total number of patients. Treatment characteristics were equally distributed between the groups. Pharmacological treatment regimens were similar in both groups with over $95 \%$ receiving aspirin and clopidogrel. Furthermore, the use of $\beta$-blocking agents and statins was high, indicating optimal medical treatment. For the complete lists of medication at admission we refer to the 30-day follow-up article [3]. The original study showed an increased rate of periprocedural MI in patients treated with immediate PCI as compared with patients with a PCI deferred for 24-48 h. Five-year follow-up data from 133 of the 142 patients could be retrieved (94\%). Three patients were lost to follow-up in the immediate group and six patients were lost to follow-up in the deferred group (Fig. 1). 


\title{
Advertisement placed here.
}

\author{
CSS Bohn

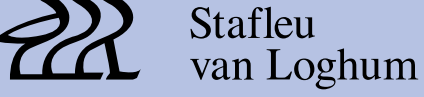 \\ Springer Media
}

Houten 2015 


\title{
Advertisement placed here.
}

\author{
CSS Bohn

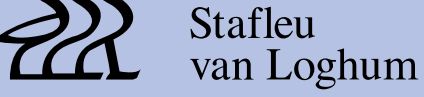 \\ Springer Media
}

Houten 2015 
Table 1 Baseline and patient characteristics

\begin{tabular}{|c|c|c|c|}
\hline Characteristics & $\begin{array}{l}\text { Immediate } \\
\text { PCI }(n=73)\end{array}$ & $\begin{array}{l}\text { Deferred } \\
\text { PCI }(n=69)\end{array}$ & $p$-value \\
\hline \multicolumn{4}{|l|}{ Demographics } \\
\hline Male sex & $51(70)$ & $51(74)$ & 0.6 \\
\hline Age (years), mean (SD) & $63(12)$ & $62(12)$ & 0.8 \\
\hline Risk factors & $41(56)$ & $34(49)$ & 0.4 \\
\hline \multicolumn{4}{|l|}{ Age $>60$ years } \\
\hline Known CAD & $27(37)$ & $25(36)$ & 0.6 \\
\hline Diabetes mellitus & $14(19)$ & $14(20)$ & 1.0 \\
\hline Hypertension & $39(53)$ & $23(33)$ & 0.03 \\
\hline Smoking & $28(38)$ & $27(39)$ & 0.8 \\
\hline Family history of IHD & $32(44)$ & $29(42)$ & 0.8 \\
\hline Hyperlipidaemia & $28(38)$ & $22(32)$ & 0.6 \\
\hline Peripheral artery disease & $5(7)$ & $3(4)$ & 0.7 \\
\hline \multicolumn{4}{|l|}{ Cardiac history } \\
\hline Previous MI & $15(21)$ & $18(26)$ & 0.5 \\
\hline Previous PCI & $20(27)$ & $13(19)$ & 0.2 \\
\hline Previous CABG & $8(11)$ & $1(1)$ & 0.02 \\
\hline Previous CHF & $1(1)$ & $1(1)$ & 0.3 \\
\hline \multicolumn{4}{|l|}{ GRACE-risk score } \\
\hline$\leq 140$ & $59(81)$ & $54(78)$ & 0.7 \\
\hline$>140$ & $14(19)$ & $15(22)$ & 0.7 \\
\hline \multicolumn{4}{|c|}{ Coronary angiographic characteristics } \\
\hline \multicolumn{4}{|c|}{ Number of diseased vessels } \\
\hline 1 & $30(41)$ & $37(54)$ & \\
\hline 2 & $33(45)$ & $22(32)$ & 0.3 \\
\hline 3 & $10(14)$ & $9(13)$ & \\
\hline \multicolumn{4}{|l|}{ TIMI flow after PCI } \\
\hline $0-2$ & $4(5)$ & $4(6)$ & 1.0 \\
\hline 3 & $69(95)$ & $64(93)$ & \\
\hline Complete revascularisation & $51(70)$ & $54(78)$ & 0.3 \\
\hline \multicolumn{4}{|l|}{ PCI characteristics } \\
\hline No PCI performed & $0(0)$ & $1(1)$ & \\
\hline Culprit lesions PCI & $73(100)$ & $68(99)$ & 0.5 \\
\hline Multiple lesions treated & $21(29)$ & $16(23)$ & 0.4 \\
\hline
\end{tabular}

Data are expressed as number (\%) unless stated otherwise.

$S D$ standard deviation, $C A D$ coronary artery disease, $M I$ myocardial infarction, $P C I$ percutaneous coronary intervention, $C A B G$ coronary artery bypass graft, $C H F$ congestive heart failure, $I H D$ ischaemic heart disease.

\section{Five-year follow-up}

No significant difference was found between the immediate and the deferred group with respect to the composite endpoint of death or spontaneous MI (17.8 vs $10.1 \%$; HR $1.55,95 \%$ CI: $0.73-4.22, p=0.21)$. Mortality at 5 years was $8.22 \%$ in the immediate group and $8.70 \%$ in the deferred group (HR 0.90, $95 \%$ CI: $0.29-2.80, p=0.86$, Fig. 2 ).

However, a significant difference was found with respect to spontaneous MI at 5-year follow-up. In total, 8 patients in the immediate group and 1 patient in the deferred group experienced a spontaneous MI (11.0 and $1.4 \%$; HR 4.46, $95 \%$ CI: $1.21-16.50, p=0.02$, Fig. 3). Since these events occurred at least 30 days after the index hospitalisation, this

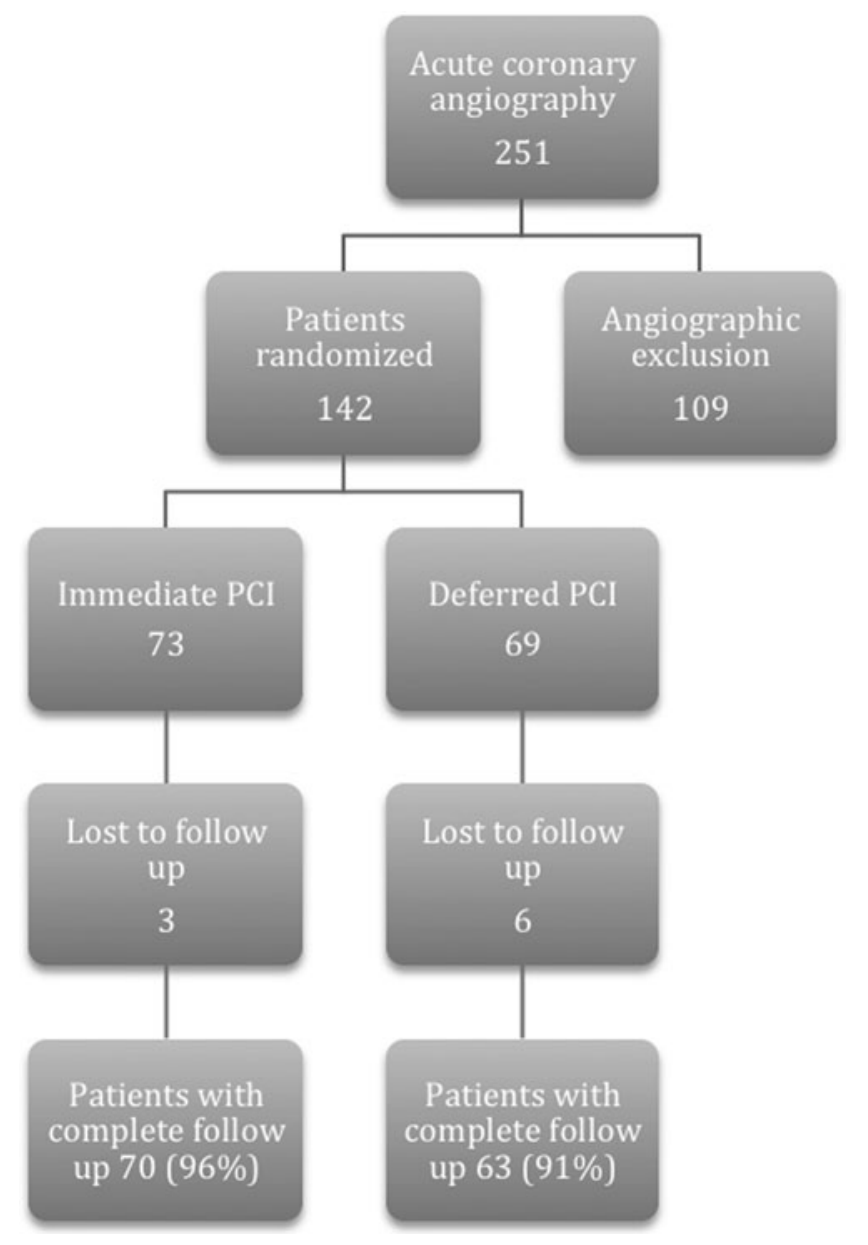

Fig. 1 The reasons for (late) angiographic exclusion $(n=109)$ were: no significant CAD: $n=55$, CABG: $n=27$, instent restenosis: $n=9$, clinically driven immediate PCI $n=8$, culprit lesion not amenable to PCI: $n=6$ : chronic total occlusion: $n=4$

difference was irrespective of initial procedure-related myocardial infarction (Table 2). A sub-group analysis showed that most of the spontaneous MIs occurred in the index vessel (Table 3). There were no significant differences in the re-PCI rate at 5-year follow-up between the immediate and deferred group. The re-PCI rate was $6.8 \%$ in the immediate group and $11.6 \%$ in the deferred group (HR $0.5395 \% \mathrm{CI}$ : $0.18-1.59, p=0.26$ ).

\section{Discussion}

After 5 years, no differences appeared in the composite endpoint of death and spontaneous MI between the groups. However, the long-term follow-up of the OPTIMA trial suggests an increased risk of a spontaneous MI in those patients treated with an immediate PCI for their index NSTE-ACS. The pathophysiology behind this excess of spontaneous MI is most likely multifactorial. 


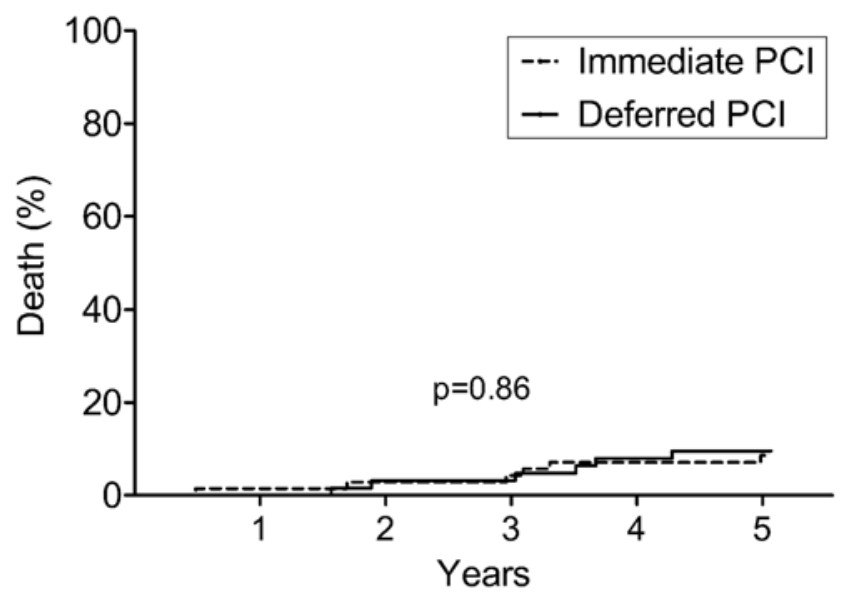

Fig. 2 All-cause death (94\% follow-up rate)

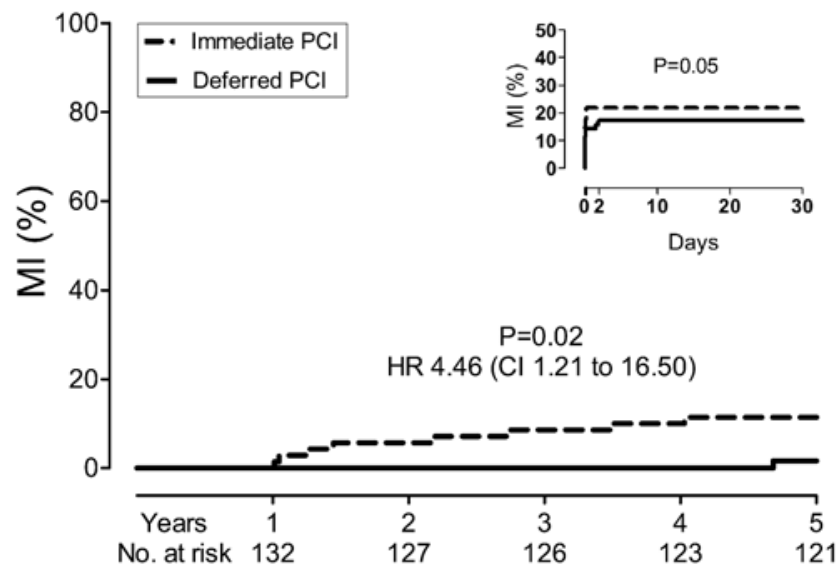

Fig. 3 Event rate filtered for the index myocardial infarction (MI) during hospitalisation is shown in the main figure. Event rate of first 30 days of follow-up are presented on the right side. $H R$ hazard ratio

NSTE-ACS represents $75 \%$ of all ACS. It has been demonstrated that routine angiography and revascularisation after NSTE-ACS reduces mortality by $25 \%$, MI by $18 \%$, and re-hospitalisation for unstable angina by $31 \%$ at midterm follow-up [8].

In the past years, several studies have evaluated the influence of the timing of intervention in patients with NSTEACS. However, comparison of data and interpretation of the results remain difficult due to methodological differences between the studies [9-14].

The OPTIMA trial is the only trial that has actually randomised the timing of administering PCI instead of the timing of angiography. This approach is the only proper way to evaluate the influence of time on PCI outcome. However, it could be remarked that randomisation after an angiography could lead to bias in the selection of patients. As such, during the OPTIMA trial, great care was taken to include intermediate to high-risk patients and not to select on angiographic features. Arguably, a large number of patients with low TIMI
Table 2 Study endpoints at 5-year follow-up

\begin{tabular}{lllll}
\hline & $\begin{array}{l}\text { Immediate } \\
n=73(\%)\end{array}$ & $\begin{array}{l}\text { Deferred } \\
n=69(\%)\end{array}$ & HR (CI) & $p$-value \\
\hline $\begin{array}{l}\text { Death or sponta- } \\
\text { neous MI }\end{array}$ & $13(17.8)$ & $7(10.1)$ & $\begin{array}{l}1.76 \\
(0.73-4.22)\end{array}$ & 0.2 \\
Death & $6(8.2)$ & $6(8.7)$ & $\begin{array}{l}0.90 \\
(0.29-2.80)\end{array}$ & 0.9 \\
& & $1(1.4)$ & $\begin{array}{l}4.46 \\
(1.21-16.5)\end{array}$ & 0.02 \\
Spontaneous MI & $8(11)$ & $\begin{array}{l}0.53 \\
(0.18-1.59)\end{array}$ & 0.3 \\
Re-PCI & $5(6.8)$ & $8(11.6)$ & &
\end{tabular}

Composite endpoint and individual endpoints.

$M I$ myocardial infarction, $P C I$ percutaneous coronary intervention, $H R$ hazard ratio.

Table 3 Characteristics of spontaneous myocardial infarction

\begin{tabular}{|c|c|c|c|c|}
\hline & $\begin{array}{l}\text { Previous } \\
\text { CABG }\end{array}$ & $\begin{array}{l}\text { Index vessel } \\
\text { Non-instent }\end{array}$ & $\begin{array}{l}\text { Index ves- } \\
\text { sel Instent } \\
\text { restenosis }\end{array}$ & $\begin{array}{l}\text { Spontaneous } \\
\text { MI non index } \\
\text { vessel related }\end{array}$ \\
\hline $\begin{array}{l}\text { Immediate } \\
n=8\end{array}$ & 2 & 2 & 2 & 4 \\
\hline $\begin{array}{l}\text { Deferred } \\
n=1\end{array}$ & - & - & 1 & - \\
\hline
\end{tabular}

flows at initial coronary angiogram were randomised [3]. The OPTIMA trial showed an increased rate of periprocedural MI in patients treated with immediate PCI. Most PCI-related infarcts were small, with little impact on myocardial function, and probably resulted from micro-emboli from the atherosclerotic plaque or disrupted thrombus particles, or formed during angioplasty or thrombotic side branch occlusions [15].

In order to decide the best treatment strategy for patients presenting with NSTE-ACS, long-term outcomes are essential. The long-term follow-up of the OPTIMA trial suggests an increased risk of spontaneous $\mathrm{MI}$ in those patients treated with an immediate PCI for their index NSTE-ACS. The long-term results show that half of the culprit lesions for spontaneous MI in the immediate group occurred within the index vessel. While the increase in re-infarction is -at least partly- explained by events originating from the index vessel, one could hypothesise a causal relationship between the timing of PCI during an acute event and the risk of a re-infarction later on.

For example, the increase in general coronary vascular tone that is often observed during an acute event could hamper the proper sizing of the stent. Incorrect sizing could lead to an increased risk of malpositioning and eventually cause a late ischaemic event [16]. Moreover, immediate PCI might have a higher risk of stenting non-significant lesions, due to overestimation of lesion severity in the very acute setting. Functional tests such as fractional flow reserve to assess the clinical significance of coronary artery stenosis of moderate/borderline severity do improve differentiation but were 
not used in the initial trial [17]. In addition, the high levels of coagulation and platelet activation that accompany an acute event are thought to increase the risk of periprocedural complications, including MI. Correspondingly, side branch occlusions and peripheral embolisation are more frequently seen in the hyper-acute setting [18-21]. While these complications increase, the long-term risks remain unknown. In accordance with a pooled analysis of three large ACS trials regarding the use of routine versus selective invasive procedures, the current study did not find a clear relation between the occurrence of periprocedural MI and the risk of late spontaneous MI or death [4]. Delaying the index procedure in order to allow for normalisation of the coronary vascular tone may result in a decrease in inflammatory, coagulation and platelet activity and might therefore be safer.

Some of the spontaneous MIs in the immediate group were not related to the index vessel. Deeper disease progression due to an imbalance in baseline characteristics such as a higher prevalence of previous CABG, hypertension and a mildly increased prevalence of dyslipidaemia in the immediate PCI group could correspond to the higher prevalence of late spontaneous MIs. There were no differences in the medical treatment post-PCI between groups; however, longterm medical compliance was not evaluated.

Whether a patient will develop an ST elevation or nonST elevation ECG during ischaemia depends on the location of the culprit lesion and on the existence and extent of the collaterals. But sometimes STEMI and NSTE-ACS are syndromes without a sharp contrast: properties overlap. IJkema et al. stated that primary PCI might be considered regardless of the nature of the ST deviation [22]. Advancing developments in the pharmacological treatment of the ACS include the use of more potent statins, new anticoagulants and more reliable and potent platelet inhibitors [23]. In addition, novel intervention techniques including the use of fractional flow rate, intravascular ultrasound and optical coherence tomography guiding, could increase the safety and effectiveness of the index procedure. Moreover, a newer generation of drug-eluting stents and bioresorbable vascular scaffolds could prove safer in terms of long-term complications. The question remains whether these developments will provide a safer medium when treating those patients with immediate PCI. Future trials, such as the OPTIMA 2 (NTR3861), are currently recruiting patients in order to address this question.

For now, there appears to be no justifiable reason to rush patients to the cathlab when they can be stabilised medically. The 'wait a day' approach might even prove safer regarding both the risk of periprocedural and long-term spontaneous MI.

\section{Study limitations}

The proposed number of patients included could not be achieved. In addition, there were 9 patients lost to follow- up. As such, the study was too underpowered to detect a difference in the clinical event rate at 5 years.

\section{Conclusion}

The OPTIMA trial showed a higher incidence of periprocedural MI in NSTE-ACS patients treated with immediate PCI, as opposed to a 'wait a day' approach. Long-term follow-up showed no difference in mortality. However, the immediate strategy seems to be accompanied by an increased risk of late spontaneous MI as well. The exact mechanisms by which this excess is caused are currently unknown, but are most likely multifactorial in origin. Given the low numbers of total recurrent ischaemic events, these findings should be interpreted as hypothesis-generating. More long-term follow-up data of earlier and larger trials that randomised to timing of angiography in NSTE-ACS are eagerly sought after.

Acknowledgments Lea Dijksman, Research Department of Onze Lieve Vrouwe Gasthuis for her supportive advice.

Funding The Netherlands Heart Foundation supported the study. Research grant number: 2003B282.

\section{Trial registration number ISRCTN80874637}

Conflict of interests None declared.

Open Access This article is distributed under the terms of the Creative Commons Attribution 4.0 International License (http://creativecommons.org/licenses/by/4.0/), which permits unrestricted use, distribution, and reproduction in any medium, provided you give appropriate credit to the original author(s) and the source, provide a link to the Creative Commons license, and indicate if changes were made.

\section{References}

1. Hamm CW, Bassand JP, Agewall S, et al. ESC Guidelines for the management of acute coronary syndromes in patients presenting without persistent ST-segment elevation: the Task Force for the management of acute coronary syndromes (ACS) in patients presenting without persistent ST-segment elevation of the European Society of Cardiology (ESC). Eur Heart J. 2011;32:2999-3054.

2. Anderson JL, Adams CD, Antman EM, et al. 2012 ACCF/AHA Focused Update Incorporated Into the ACCF/AHA 2007 Guidelines for the Management of Patients With Unstable Angina/Non-ST-Elevation Myocardial Infarction: a Report of the American College of Cardiology Foundation/American Heart Association Task Force on Practice Guidelines. J Am Coll Cardiol. 2013;61:179-347.

3. Riezebos RK, Ronner E, Ter Bals E, et al. Immediate versus deferred coronary angioplasty in non-ST-segment elevation acute coronary syndromes. Heart. 2009;95:807-12.

4. Damman P, Wallentin L, Fox KA, et al. Long-term cardiovascular mortality after procedure-related or spontaneous myocardial infarction in patients with non-ST-segment elevation acute coronary syndrome: a collaborative analysis of individual patient data from the FRISC II, ICTUS, and RITA-3 trials (FIR). Circulation. 2012;125:568-76. 
5. Damman P, Clayton T, Wallentin L, et al. Effects of age on longterm outcomes after a routine invasive or selective invasive strategy in patients presenting with non-ST segment elevation acute coronary syndromes: a collaborative analysis of individual data from the FRISC II-IC. Heart. 2012;98:207-13.

6. Damman P, Hirsch A, Windhausen F, et al. 5-year clinical outcomes in the ICTUS (Invasive versus Conservative Treatment in Unstable coronary Syndromes) trial a randomized comparison of an early invasive versus selective invasive management in patients with non-ST-segment elevation acute coronary syndrome. J Am Coll Cardiol. 2010;55:858-64.

7. Thygeses K, Alpert JS, Jaffe AS, et al. Third universal definition of myocardial infarction. Circulation. 2012;126:2020-35.

8. Bavry AA, Kumbhani DJ, Rassi AN, et al. Benefit of early invasive therapy in acute coronary syndromes: a meta-analysis of contemporary randomized clinical trials. J Am Coll Cardiol. 2006;48:1319-25.

9. Riezebos RK, Verheugt FW. Timing of angiography in non-ST elevation myocardial infarction. Heart. 2013;99:1867-73.

10. Mehta SR, Granger CB, Boden WE, et al. Early versus delayed invasive intervention in acute coronary syndromes. N Engl J Med. 2009;360:2165-75.

11. Montalescot G, Cayla G, Collet JP, et al. Immediate vs delayed intervention for acute coronary syndromes: a randomized clinical trial. JAMA. 2009;302:947-54.

12. Mills NL, Churchhouse AM, Lee KK, et al. Implementation of a sensitive troponin I assay and risk of recurrent myocardial infarction and death in patients with suspected acute coronary syndrome. JAMA. 2011;305:1210-6.

13. Thiele H, Rach J, Klein N, et al. Optimal timing of invasive angiography in stable non-ST-elevation myocardial infarction: the Leipzig Immediate versus early and late PercutaneouS coronary Intervention triAl in NSTEMI (LIPSIA-NSTEMI Trial). Eur Heart J. 2012;33:2035-43.
14. Neumann FJ, Kastrati A, Pogatsa-Murray G, et al. Evaluation of prolonged antithrombotic pretreatment ("cooling-off" strategy) before intervention in patients with unstable coronary syndromes: a randomized controlled trial. JAMA. 2003;290:1593-9.

15. Califf RM, Abdelmeguid AE, Kuntz RE, et al. Myonecrosis after revascularization procedures. J Am Coll Cardiol. 1998;31:241-51.

16. Attizzani GF, Capodanno D, Ohno Y, et al. Mechanisms, pathophysiology, and clinical aspects of incomplete stent apposition. J Am Coll Cardiol. 2014;63:1355-67.

17. De Bruyne B, Fearon WF, Pijls NH, et al. Fractional flow reserveguided PCI for stable coronary artery disease. N Engl J Med. 2014;371:1208-17.

18. Babu GG, Walker JM, Yellon DM, et al. Peri-procedural myocardial injury during percutaneous coronary intervention: an important target for cardioprotection. Eur Heart J. 2011;32:23-31.

19. Herrmann J. Peri-procedural myocardial injury: 2005 update. Eur Heart J. 2005;26:2493-519.

20. Ronner E, Boersma E, Laarman GJ, et al. Early angioplasty in acute coronary syndromes without persistent ST-segment elevation improves outcome but increases the need for six-month repeat revascularization: an analysis of the PURSUIT Trial. Platelet glycoprotein IIB/IIIA in Unstable angina: receptor Suppression Using Integrilin Therapy. J Am Coll Cardiol. 2002;39:1924-9.

21. Kereiakes DJ, Gurbel PA. Peri-procedural platelet function and platelet inhibition in percutaneous coronary intervention. JACC Cardiovasc Interv. 2008; 1:111-21.

22. IJkema BB, Bonnier JJ, Schoors D, et al. Role of the ECG in initial acute coronary syndrome triage: primary PCI regardless presence of ST elevation or of non-ST elevation. Neth Heart J. 2014;22:484-90.

23. Yetgin T, Linden MM van der, Vries AG de, et al. Adoption of prasugrel into routine practice: rationale and design of the Rijnmond Collective Cardiology Research (CCR) study in percutaneous coronary intervention for acute coronary syndromes. Neth Heart J. 2014;22:55-61.

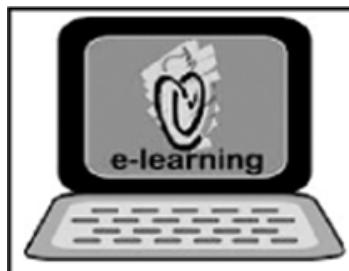

\section{CVOI E-learning formula!}

This is the CVOI e-learning article. The author has prepared 10 questions which are available through the website of the Cardiovascular Educational Institute (CVOI). Please follow the instructions below.

After finishing the questions you will be asked to fill in your name, hospital and e-mail address; then press the button 'verzenden'.

When 6 out of the 10 questions are answered correctly, you acquire 1 accreditation point granted by the Quality Committee of the Netherlands Society of Cardiology (NVVC). The acquired point will be credited to your personal file in the GAIA system. You will also receive an e-mail with all the correct answers.

Over a period of one year 10 e-learning articles will appear in 10 subsequent NHJ editions. In each edition the e-learning article will be recognisable by a special icon. On an annual basis you can collect 10 accreditation points. The accreditation points are credited in the GAIA system by the CVOI.

If you need additional information, please contact the CVOI by e-mail: cvoi@cvoi.org or by phone: 030-2345001.

E.E. van der Wall

Chief editor NHJ
K.B. Schick

Coordinator CVOI 